\title{
Annexure 1
}

\section{Program for finding the minimum of the objective function}

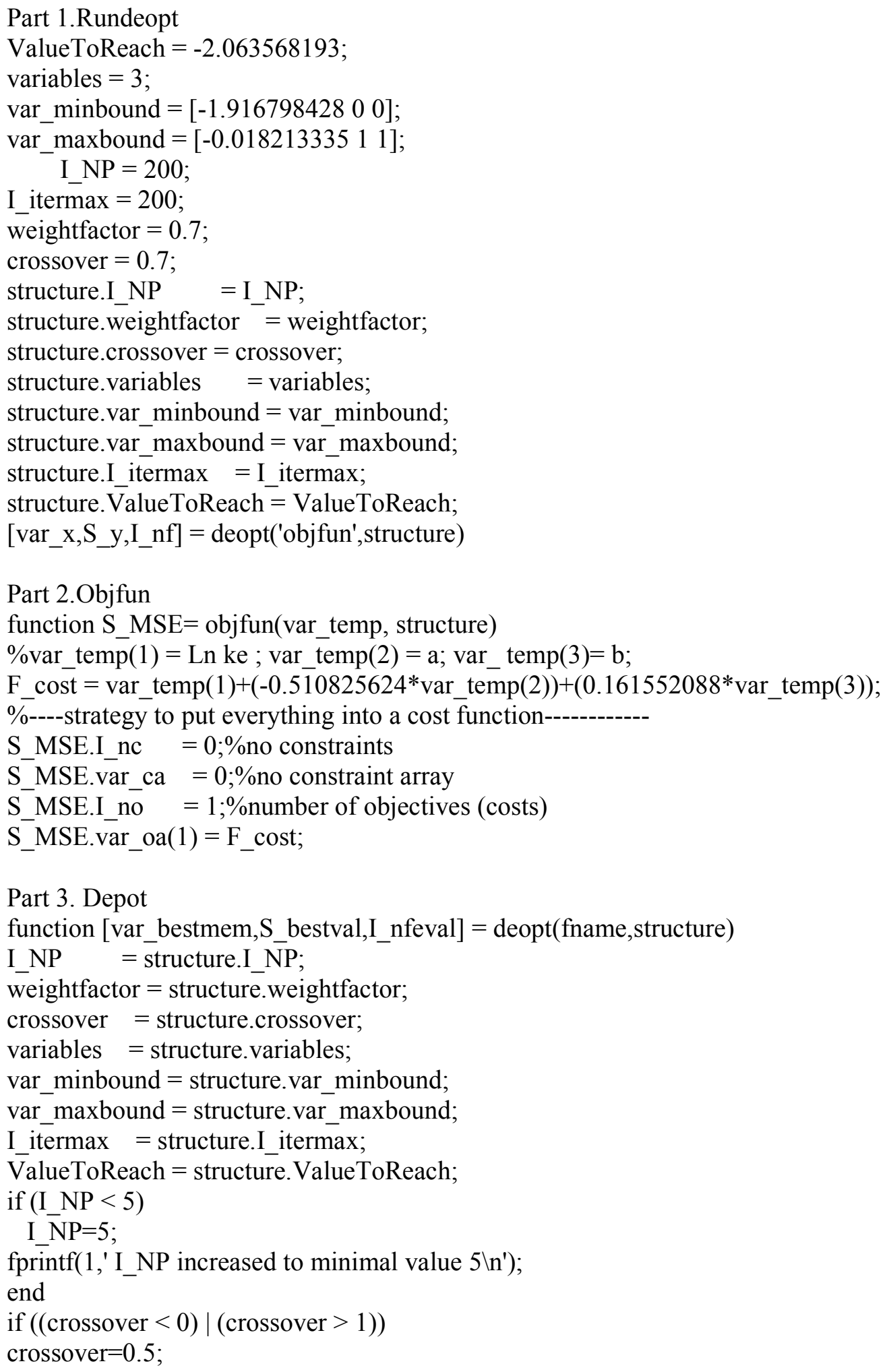

Part 3. Depot

function [var_bestmem,S_bestval,I_nfeval] = deopt(fname,structure) I_NP $=\overline{\text { structure.I_NP; }}$

weightfactor $=$ structure. weightfactor;

crossover = structure.crossover;

variables = structure.variables;

var_minbound $=$ structure.var_minbound;

var_maxbound $=$ structure.var_maxbound;

I_itermax = structure.I_itermax;

$\overline{\text { ValueToReach }}=$ structure. ValueToReach;

if (I_NP < 5)

I $\mathrm{NP}=5$;

fprintf(1,' I_NP increased to minimal value $5 \backslash n$ ');

end

if $(($ crossover $<0) \mid($ crossover $>1))$

crossover $=0.5$; 
fprintf(1,'crossover should be from interval $[0,1]$; set to default value $0.5 \backslash n$ ');

end

if (I_itermax $<=0$ )

I_itermax $=200$;

fprintf(1,'I_itermax should be >0; set to default value $\left.200 \backslash n^{\prime}\right)$;

end

FM_pop $=$ zeros(I_NP, variables);

for $\mathrm{k}=1: \mathrm{I} \mathrm{NP}$

FM_pop $(\bar{k},:)=$ var_minbound $+\operatorname{rand}(1$, variables $) . *($ var_maxbound - var_minbound $)$;

end

FM_popold = zeros(size(FM_pop));

var_bestmem = zeros(1, variables);

var_bestmemit $=$ zeros $(1$, variables $)$;

I_nfeval $=0$;

I_best index $=1$;

S_val(1) = feval(fname,FM_pop(I_best_index,:), structure);

S_bestval $=$ S_val $(1)$;

I_nfeval =I_nfeval +1 ;

for $\mathrm{k}=2: \mathrm{I}$ NP

S_val(k) $=$ feval(fname,FM_pop(k,:),structure);

I_nfeval =I nfeval + 1;

if $\left(\right.$ left_win $\left.\overline{\left.\text { S_val }(k), S \_b e s t v a l\right)}==1\right)$

I_best_index $=\mathrm{k}$;

S_bestval = S_val $(\mathrm{k})$;

end

end

var_bestmemit $=$ FM_pop(I_best_index,:);

var_bestmem = var_bestmemit;

I iter $=1$;

while ((I_iter $<$ I_itermax) \& (S_bestval.var_oa(1) $>$ ValueToReach)

FM_popold $=$ FM_pop;

structure.FM_pop = FM_pop;

structure.var_bestmem = var_bestmem;

var_ind $=$ randperm(4);

var_al =randperm(I_NP);

var_rt $=$ rem(var_rot+var_ind(1),I_NP);

var_a2 = var_a1 (var_rt+1);

var_rt $=$ rem(var_rot+var_ind(2),I_NP);

var_a3 = var_a 2 (var_rt+1)

var_ert $=$ rem(var_rot+var_ind(3),I_NP);

var_a4 = var_a $3($ var_rt+1)

var_ $\mathrm{rt}=$ rem(var_rot+var_ind(4),I_NP);

var_a5 = var_a $\overline{4}($ var_rt+1)

FM_pm $1=\overline{F M}$ _popold(var_a1,:);

FM_pm2 = FM_popold(var_a2,:);

FM_pm3 = FM_popold(var_a3,:);

for $\mathrm{k}=1: \mathrm{I} \_\mathrm{NP}$

FM_bm $(\bar{k},:)=$ var_bestmemit;

end

FM_mui $=$ rand(I_NP,variables $)<$ crossover; 
FM_mpo $=$ FM_mui $<0.5$;

FM_ui $=F M \_-\overline{p m} 3+$ weightfactor*(FM_pm1 - FM_pm2);

$\mathrm{FM} \_u i=\mathrm{FM} \_$popold. $* \mathrm{FM}$ _mpo $+\mathrm{FM}$ _ui.*FM_mui;

FM_origin $=F M \_p m 3$;

for $\overline{\mathrm{k}}=1: \mathrm{I}$ NP

for $\mathrm{j}=1$ :variables

if (FM_ui (k,j) > var_maxbound(j))

FM_ui(k,j) $=$ var_maxbound $(j)+$ rand $\left(F M \_o r i g i n(k, j) ~-~ v a r \_m a x b o u n d(j)\right)$;

end

if (FM_ui(k,j) <var_minbound(j))

FM_ui(k,j) $=$ var_minbound $(\mathrm{j})+\operatorname{rand} *\left(F M \_o r i g i n(k, j)-v a r \_m i n b o u n d(j)\right)$;

end

end

S_tempval = feval(fname,FM_ui(k,:), structure);

I_nfeval =I_nfeval + 1;

if $($ left_win $(\bar{S}$ _tempval,S_val $(\mathrm{k}))==1)$

FM_pop $(\mathrm{k},:)=\mathrm{FM} \_$ui $(\mathrm{k},:)$;

S_val(k) = S_tempval;

if $($ left_win(S_tempval,S_bestval) $==1$ )

S_bestval $=$ S_tempval;

var_bestmem $=$ FM_ui $(\mathrm{k},:$ );

end

end

end

var_bestmemit $=$ var_bestmem;

I_iter $=$ I_iter +1 ;

end

Part 4.Letfwin

functionI_z $=$ left_win(S_x,S_y);

I_z $=1$;

if $\left(\mathrm{S} \_x . I \_n c>0\right)$

for $\mathrm{k}=1: \overline{\mathrm{S}}$ _x.I_nc

if $($ S_x.var_ca( $(\mathrm{k})>0)$

if $\left(S_{-} \_\right.$x.var_ca(k) $>$S_y.var_ca(k))

I_ $\mathrm{z}=0$;

end

end

end

end

if (S_x.I_no $>0)$

for $\mathrm{k}=1: \bar{S}$ x.I_no

if (S_x.var_oa $(\mathrm{k})>\mathrm{S} \_$y.var_oa $\left.(\mathrm{k})\right)$

I_ $\mathrm{z}=0$;

end

end

end 
